\title{
Molecular Imaging of Inflammation: Current Status
}

\author{
Dima A. Hammoud \\ Center for Infectious Disease Imaging, Radiology and Imaging Sciences, National Institutes of Health, Bethesda, Maryland
}

The ability to image inflammation in vivo can improve our understanding of the pathophysiology underlying various disease etiologies, including cancer, atherosclerosis, and neurodegeneration. A great wealth of preclinical and translational research has been and is currently being developed to decipher the involvement of the immune system in disease pathophysiology, quantify the course of a disease, and visualize the potential detrimental effects of excessive inflammation. Down the road, the ultimate goal is to have clinical noninvasive in vivo imaging biomarkers of inflammation that will help diagnose disease, establish prognosis, and gauge response to preventative and therapeutic strategies.

Key Words: inflammation; molecular imaging; PET; MRI; iron oxide nanoparticles

J Nucl Med 2016; 57:1161-1165

DOI: 10.2967/jnumed.115.161182

Imaging of tissue inflammation using conventional methods such as CT and MRI provides information mainly about structural changes in the involved tissues. Those changes can include edema (accumulation of fluid in the extracellular space), contrast enhancement (endothelial disruption), and organ damage. On the other hand, nuclear medicine techniques such as radiolabeled white blood cells and ${ }^{18} \mathrm{~F}$-FDG provide functional information about the inflammatory reaction, based on chemotaxis and glucose metabolism, respectively. More recently, more specific imaging of the inflammatory reaction has been sought, using various molecular imaging techniques, mainly PET, SPECT, and MRI. Those attempts are generally based on targeting specific elements of the immune system (e.g., macrophages or lymphocytes) but are often tailored differently for various organs, based on the organs' individual properties.

\section{TARGETING OF THE IMMUNE SYSTEM}

One way of imaging inflammation is by targeting the upregulation and trafficking of immune system cells as they

Received Mar. 30, 2016; revision accepted May 11, 2016.

For correspondence or reprints contact: Dima A. Hammoud, National Institutes of Health/Clinical Center, 10 Center Dr., Room 1C368, Bethesda, MD 20814-9692.

E-mail: hammoudd@cc.nih.gov

Published online May 12, 2016.

COPYRIGHT (C 2016 by the Society of Nuclear Medicine and Molecular Imaging, Inc.

interact with noxious stimuli, whether infectious, autoimmune, or degenerative. For many years, targeting of immune cells was mostly isotope-based, with SPECT and PET applications. More recently, MRI-based approaches have gained momentum, especially with the advent of different types of magnetic nanoparticles that can be functionalized for specific targeting of surface markers and other cell constituents. The accumulation of magnetic nanoparticles results in shortening of the $\mathrm{T} 2$ and $\mathrm{T} 2 *$ relaxation times of surrounding tissues, which causes signal reduction (negative contrast) on MR images. A previously published article (1) provides more information about the physics of detecting paramagnetic nanoparticles in vivo.

\section{Imaging of Macrophages and Monocytes}

In the innate response of the immune system to injury or pathogens, macrophages and monocytes have an important and diverse role that includes antigen presentation (to lymphocytes), cytokine and chemokine secretion (pro- and antiinflammatory), and, most prominently, phagocytosis (2).

Monocytes and macrophages have been targeted in the setting of inflammation using a variety of nanomaterials, such as the small and ultrasmall paramagnetic iron oxide nanoparticles (SPIO and USPIO, respectively) (3). The phagocytic abilities of the monocyte-macrophage system are generally assumed to account for the process of nanoparticle uptake (4). This process depends on many factors, the most important of which are the size and shape of the nanoparticles, the characteristics of their surface coating, and opsonization. By modifying their size $(<$ or $>100 \mathrm{~nm})$, charge (positive vs. negative), and surface coating (glycine increases uptake by activated macrophages) and adding specific targeting ligands (e.g., macrophage receptors such as $\mathrm{C}-\mathrm{C}$ chemokine receptor 2 and $\mathrm{CX} 3 \mathrm{C}$ chemokine receptor 1 ), the degree of uptake and, potentially, the specific population uptake can be regulated (4).

Macrophages can also be imaged using PET and SPECT ligands (e.g., modified dextran nanoparticles labeled with ${ }^{89} \mathrm{Zr}$ ), optical imaging (quantum dots and fluorochromelabeled nanoparticles), and CT. An excellent review on this topic has been previously published (4).

\section{Imaging of Lymphocytes}

B lymphocytes play a major role in the humoral-immunity component of the adaptive immune system by producing 
antibodies in response to various antigens. Multiple subtypes of B cells are generally identified by flow cytometry through specific cellular markers. B-cell dysfunction and dysregulation, on the other hand, can lead to various autoimmune diseases, making those pathogenic B cells the main targets for corresponding therapeutic approaches. Imaging biomarkers can be developed on the basis of those therapies. Perhaps the best example is the use of radiolabeled rituximab, an anti-CD20 antibody. ${ }^{99 \mathrm{~m}} \mathrm{Tc}$ - and ${ }^{124} \mathrm{I}$-radiolabeled rituximab has been successfully used in patients with diseases such as rheumatoid arthritis, psoriatic arthritis, and sarcoidosis (5). The limitations of this approach include the potential for immunogenicity and sequestration within the spleen, which might require preadministration of unlabeled rituximab (6). A discussion of radioimmunotherapies using other types of humanized B-cell antibodies, such as yttrium-labeled ibritumomab tiuxetan and epratuzumab tetraxetan, is beyond the scope of this article.

$\mathrm{T}$ lymphocytes play an important role in cell-mediated immune response. Various cellular markers are responsible for activating the T-cell population in response to antigen presentation. Imaging of T lymphocytes thus becomes important both for delineating inflammatory abnormalities and for monitoring autoimmune disorders associated with their dysregulation. Longstanding attempts at labeling $\mathrm{T}$ cells with isotopes such as ${ }^{111} \mathrm{In},{ }^{51} \mathrm{Cr}$, and ${ }^{99 \mathrm{~m}} \mathrm{Tc}$ were evaluated mostly in preclinical models of disease and achieved various levels of success but with some reported problems, such as the negative effect of certain labels_- ${ }^{99 \mathrm{~m}} \mathrm{Tc}$, for example_-on cell trafficking and migration (7). More recently, labeling with PET isotopes such as ${ }^{64} \mathrm{Cu}$ and ${ }^{18} \mathrm{~F}$ has been attempted, including labeling with ${ }^{18} \mathrm{~F}$-FDG, ${ }^{18} \mathrm{~F}$-FBEM (4-fluorobenzamido- $N$ ethylaminomaleimide), and ${ }^{64} \mathrm{Cu}$-PTSM (pyruvaldehydebis(N4-methylthiosemicarbazone) (7). Successful reporter gene labeling has been described recently as well, and a comparison between different reporter gene systems showed that the combination of a human norepinephrine transporter and a ${ }^{18} \mathrm{~F}$-fluorobenzylguanidine reporter system is the most sensitive for detection of lymphocytes in a mouse model (8). In contrast to the in vitro approach to labeling $\mathrm{T}$ lymphocytes, an alternative approach is to target these cells in vivo with labeled cytokines (e.g., IL-2, IL-12, IL-1, and IL-1ra) or chemokines (e.g., IL-8). Most of those experiments have been on preclinical models of cancer, infection, and inflammation, with the main human applications being the use of radiolabeled IL-8 and IL-2 (7). Finally, extensive probing of radiolabeled antibodies to surface markers of $\mathrm{T}$ lymphocytes such as CD25, CD3, CD4, CD45, CD5, and CD2 has been done since the 1980s, generally in animal models and patients with hematopoietic malignancies and lung cancer, with only a few applications targeting inflammatory entities such as rheumatoid arthritis (9). The limitations to this approach are not specific to this type of antibody but rather are common to most antibody-based approaches and include delayed imaging requirements due to long plasma half-lives and the potential development of human antimurine antibodies (10). A recent paper described the novel use of cys-diabodies (bivalent

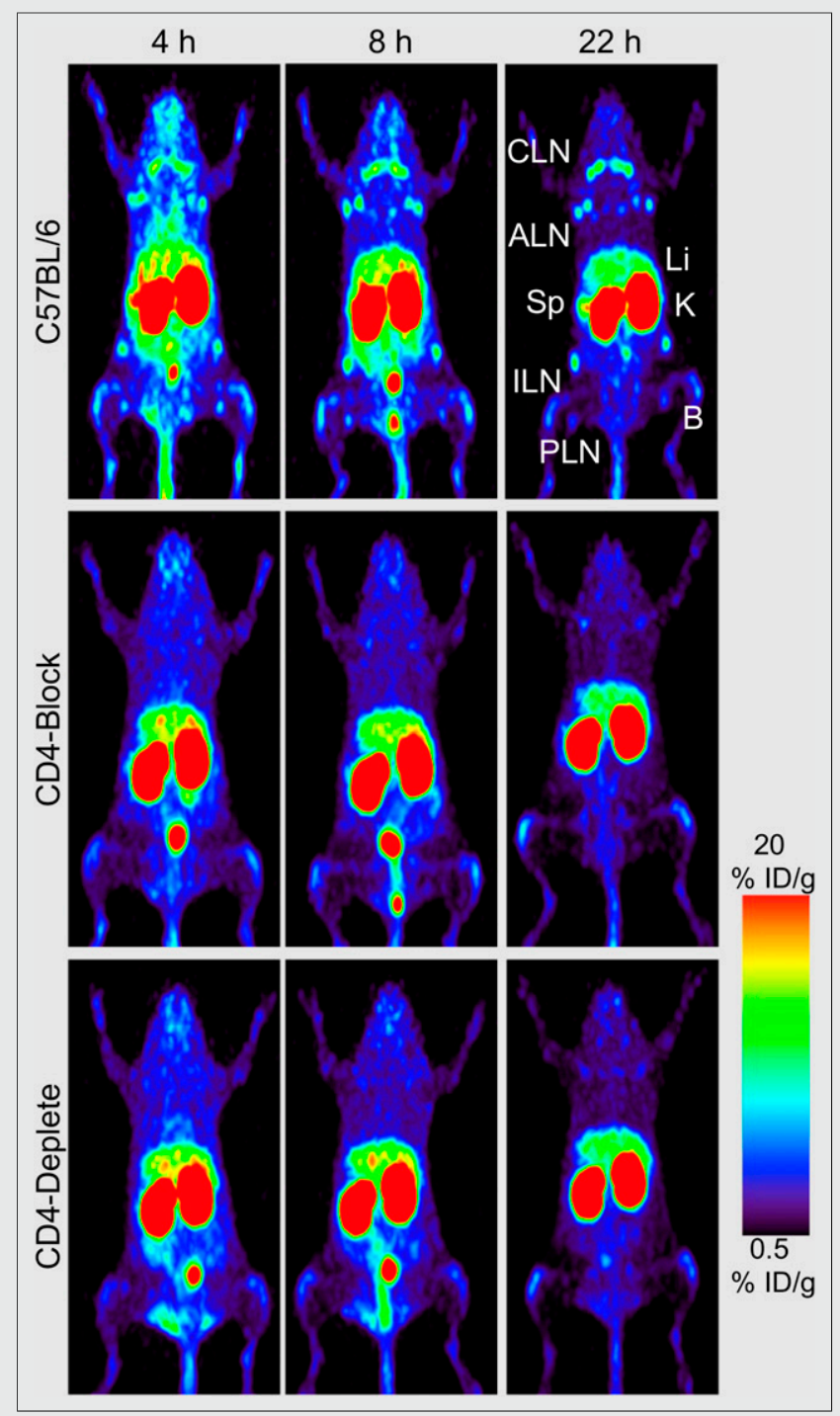

FIGURE 1. Anti-CD4 small-animal PET imaging (using ${ }^{89} \mathrm{Zr}$-malDFO-GK1.5 cys-diabodies, where malDFO is $\mathrm{N}$ (3,11,14,22,25,33-hexaoxo-4,10,15,21,26, 32-hexaaaza-10,21,32trihydroxytetratriacontane) maleimide) of T-lymphocytes in C57BL/6 wild-type, CD4-blocked, and CD4-depleted mice at 4, 8 , and $22 \mathrm{~h}$ after injection. Images are $25-\mathrm{mm}$ maximum-intensity projections. Compared with wild-type mice, both CD4-blocked and CD4-depleted animals lack uptake in axillary lymph nodes (ALN), cervical lymph nodes (CLN), inguinal lymph nodes (ILN), popliteal lymph nodes (PLN), and spleen (Sp). B = bone; ID = injected dose; K = kidney; Li = liver. (Adapted from (11).)

antibody fragments with a shorter serum half-life) against CD4 and CD8 for the visualization of lymph nodes and spleen in mice (Fig. 1) (11). An excellent review paper (7) provides more information about the imaging of $\mathrm{T}$ lymphocytes.

\section{IMAGING OF INFLAMMATION AT THE ORGAN LEVEL}

\section{Central Nervous System Inflammation}

Neuroinflammation is a suspected culprit in many diseases, including chronic infections (e.g., HIV), neurodegenerative diseases (e.g., Parkinson and Alzheimer dementia), 


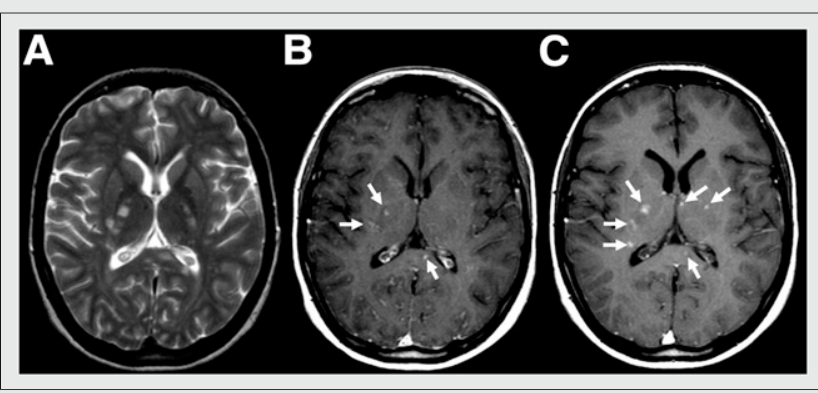

FIGURE 2. Axial MR images of inflammation in brain of young woman with multiple sclerosis. (A) Unenhanced T2-weighted image shows multiple hyperintense lesions. (B) Gadoliniumenhanced T1-weighted image before injection of USPIO shows 3 enhancing lesions (arrows). (C) At 24-48 h after injection, the original 3 lesions are seen along with 3 additional lesions (arrows). (Adapted with permission of (20).)

brain tumors (including primary and metastatic disease), and psychiatric diseases (e.g., depression, anxiety, and schizophrenia). Currently, the most common approach to neuroinflammation imaging is through targeting the translocator protein (TSPO), an outer mitochondrial membrane receptor that becomes upregulated in activated microglia, astrocytes, and blood-derived macrophages in response to central nervous system injury (12). The classic ligand is PK11195 $\left({ }^{11} \mathrm{C}-N\right.$-butan-2-yl-1-(2-chlorophenyl)- $N$-methylisoquinoline-3-carboxamide); however, a multitude of newer ligands designed to overcome the limitations of PK11195 (high lipophilicity resulting in decreased bioavailability, high nonspecific binding, and short half-life of ${ }^{11} \mathrm{C}$ ) has recently been developed (12). Along with the improved binding characteristics of the second-generation ligands, however, new problems emerged. The most important problem is a polymorphism (rs6971) located in exon 4 of the TSPO gene that is believed to result in nonconservative amino-acid substitution at position 147 from alanine to threonine (Ala147Thr) in the fifth transmembrane domain of the TSPO protein (13). This polymorphism results in 3 levels of binding, requiring, in most cases, the genotyping of subjects before quantitative assessments of TSPO density using PET. Another problem in nonfocal brain diseases is the lack of a reference region due to diffuse involvement of the brain. There have recently been encouraging descriptions of TSPO ligands that are not sensitive to polymorphism (14). Review articles about TSPO imaging in neuroinflammation have been published (12).

Other potential targets for neuroinflammation imaging are currently being evaluated, namely the cannabinoid receptors $\mathrm{CB} 1$ and $\mathrm{CB} 2$, which together constitute the endocannabinoid system (15). Multiple ligands targeting CB2 are being developed (16). Animal applications in neuroinflammatory disease models are encouraging (16). Alternative imaging approaches to neuroinflammation include targeting the arachidonic acid cascade (17) and cyclooxygenase-2 expression (18).

Neuroinflammatory changes have been targeted with iron oxide magnetic nanoparticles in animal studies of ischemia, but in human studies no correlation has been found between iron oxide-based enhancement and infarct size (19). Imaging of multiple sclerosis with magnetic nanoparticles, on the other hand, has identified more active lesions than are found by gadolinium enhancement alone. Interestingly, the most USPIO-positive lesions show no concomitant gadoliniumdiethylenetriamine pentaacetic acid (DTPA) enhancement, indicating that more severe disease is hiding behind the intact or repaired blood-brain barrier (Fig. 2) (20). Enhancement with both types of contrast agent (paramagnetic nanoparticles and gadolinium-DTPA), compared with only one or the other, reflects more aggressive lesions (20).

\section{Vascular Diseases and Atherosclerosis}

Imaging of the inflammatory component of atherosclerosis has become a major target in the last couple of decades because of the relationship that has been discovered between inflammation (macrophage activity) and the risk of plaque development and rupture (vulnerable plaque) (21). An extensive body of literature, with excellent review articles (22), is available on the use of ${ }^{18} \mathrm{~F}$-FDG PET imaging as a marker of macrophage infiltration to identify inflammatory vascular plaques. In one study, ${ }^{18} \mathrm{~F}$-FDG uptake was found to correlate positively with macrophage density (23). TSPO ligands such as ${ }^{125}$ I-DPA-713 ( $N, N$-diethyl-2-(4-methoxyphenyl)5,7-dimethylpyrazolo[1,5-a]pyrimidine-3-acetamide) (24) and ${ }^{11} \mathrm{C}-\mathrm{PK} 11195(25)$, as well as labeled DOTATATE $\left({ }^{68} \mathrm{Ga}\right.$ and ${ }^{64} \mathrm{Cu}$ ) (Fig. 3) (26), have also been used in the evaluation of plaques. Although the former ligands target TSPO expression in the infiltrative macrophages that are present in vulnerable plaques, the latter (labeled DOTATATE) seems to target the same cell population (macrophages) but through a different cellular component, the somatostatin receptor subtype 2 (26). Still at the preclinical levels, ${ }^{18}$ F-labeling of anti-VCAM1 Nanobodies seems to show promise in ApoE-deficient mice (27).

SPIO nanoparticles have been used both in animal models of atherosclerosis and in patients. The magnitude of the reduction in T2 signal intensity on high-resolution MRI after administration of superparamagnetic nanoparticles

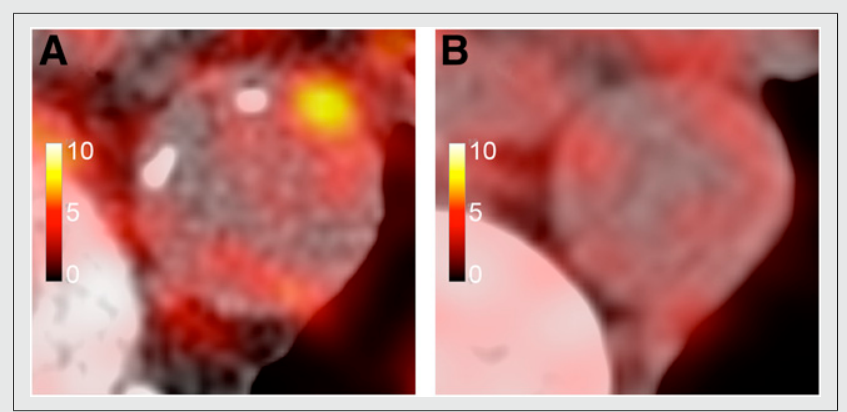

FIGURE 3. ${ }^{64} \mathrm{Cu}$-DOTATATE PET images of patient with Framingham risk score of $30(\mathrm{~A})$ and patient with Framingham risk score of 2 (B). High focal uptake is seen in thoracic aorta of first patient, compared with lower and more diffuse uptake in second patient. (Adapted from (26).) 


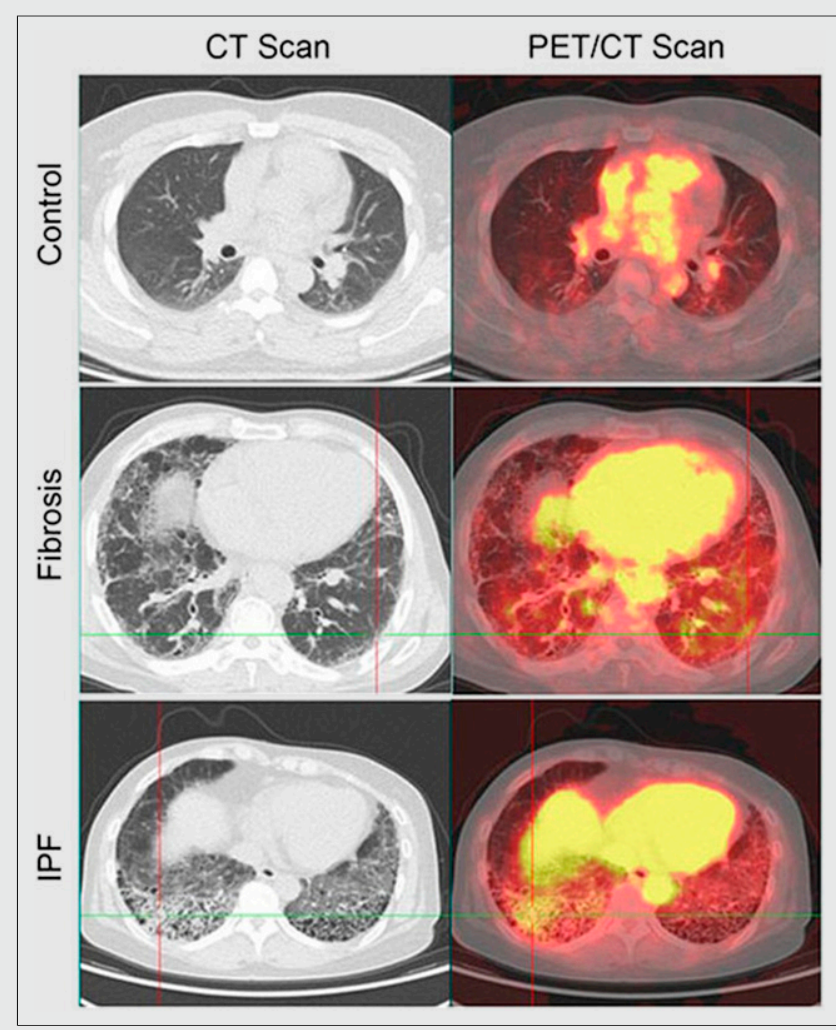

FIGURE 4. PET/CT imaging of lung inflammation. ${ }^{68} \mathrm{Ga}-\mathrm{BMV} 101$ $\mathrm{PET} / \mathrm{CT}$ probe (targeting cysteine cathepsins, which are highly expressed in activated macrophages) was injected intravenously, and images were collected $2.5 \mathrm{~h}$ later. Patient with idiopathic pulmonary fibrosis (IPF) shows increased accumulation in fibrotic regions, but patient with unclassified fibrosis shows no significant increase compared with normal-lung control. (Adapted with permission of (35).)

can theoretically quantify the macrophage burden in plaque, allowing for better characterization of the plaque and monitoring for therapy-mediated changes (28).

When ${ }^{18} \mathrm{~F}-\mathrm{FDG}$ PET and USPIO were used together in a rabbit model of atherosclerosis imaged on a combined PET/ MRI scanner, ${ }^{18} \mathrm{~F}$-FDG PET was found to be more sensitive than USPIO for the detection of early changes in plaque inflammation, although correlations between mean SUV or change in $\mathrm{T} 2 *$ relaxation rate $\left(R^{2 *}\right)$ and macrophage density (RAM-11 staining) were good (29). An excellent review article provides a detailed in-depth description of nanoparticle use in the imaging of cardiovascular disease (30).

\section{Chest, Abdominal Organs, and Joints}

Imaging of inflammation in the lungs and various abdominal organs has also been done. Here, as for most other targets, ${ }^{18} \mathrm{~F}$-FDG PET was found to be useful for imaging of postradiation pneumonitis (31), pulmonary complications of cystic fibrosis (32), and allergen-induced asthma (33). More specific than ${ }^{18} \mathrm{~F}-\mathrm{FDG}$, a novel ${ }^{18} \mathrm{~F}$-labeled tracer that binds to inducible nitric oxide synthase was recently found to accumulate in subjects with endotoxin-induced acute lung inflammation (34). Also of interest is a recent description of cysteine cathepsin- targeted imaging probes that can be used to monitor the contribution of macrophages to fibrotic disease progression in a murine model of pulmonary fibrosis and in patients with idiopathic pulmonary fibrosis (Fig. 4) (35). Cathepsins are known to be highly expressed in activated macrophages (35).

In the setting of myocardial infarction, the accumulation of USPIOs over time, as seen in one study (36), suggested progressive recruitment of inflammatory cells. This, however, remains of uncertain clinical significance. Similarly, another study (37) has shown that accumulation of USPIOs in experimental septic arthritis allows the identification of early synovitis and therapy monitoring. In addition, in a review of the role of ${ }^{18} \mathrm{~F}-\mathrm{FDG}$ in evaluating peripheral inflammatory arthritis, Bruijnen et al. (38) concluded that although such use is promising, more imaging in larger cohorts is necessary before clinical application. On the other hand, in a study comparing MRI with ${ }^{11} \mathrm{C}$-PK11195 PET to identify joint inflammation in rheumatoid arthritis patients before clinical symptoms appeared (39), the latter method was found to be more predictive of the development of clinical flare than MRI. Finally, a study on imaging renal inflammation in animals and humans $(40)$ showed that the use of ${ }^{18} \mathrm{~F}-\mathrm{FDG}$ PET and USPIO was generally able to identify regions of macrophage infiltration and its extent, especially in the setting of autoimmune diseases such as systemic lupus erythematosus and Sjögren syndrome.

\section{CONCLUSION}

Molecular imaging of inflammation is a rapidly growing field with multiple applications in a vast array of disciplines. The use of both MR-based and nuclear medicine techniques will certainly add to our understanding of the role of inflammation in cancer, neurodegeneration, infection, and psychiatric diseases. More targets, and corresponding ligands, within the immune system and various organs need to be established for the field to reach its potential.

\section{DISCLOSURE}

No potential conflict of interest relevant to this article was reported.

\section{REFERENCES}

1. Ittrich H, Peldschus K, Raabe N, Kaul M, Adam G. Superparamagnetic iron oxide nanoparticles in biomedicine: applications and developments in diagnostics and therapy. Rofo. 2013;185:1149-1166.

2. Rua R, McGavern DB. Elucidation of monocyte/macrophage dynamics and function by intravital imaging. J Leukoc Biol. 2015;98:319-332.

3. Neuwelt A, Sidhu N, Hu CA, Mlady G, Eberhardt SC, Sillerud LO. Iron-based superparamagnetic nanoparticle contrast agents for MRI of infection and inflammation. AJR. 2015;204:W302-W313.

4. Weissleder R, Nahrendorf M, Pittet MJ. Imaging macrophages with nanoparticles. Nat Mater. 2014;13:125-138.

5. Malviya G, Anzola KL, Podesta E, et al. ${ }^{99 \mathrm{~m} T c-l a b e l e d ~ r i t u x i m a b ~ f o r ~ i m a g i n g ~ B ~}$ lymphocyte infiltration in inflammatory autoimmune disease patients. Mol Imaging Biol. 2012;14:637-646.

6. Tran L, Huitema $\mathrm{AD}$, van Rijswijk MH, et al. CD20 antigen imaging with ${ }^{124} \mathrm{I}-$ rituximab PET/CT in patients with rheumatoid arthritis. Hum Antibodies. 2011;20: 29-35. 
7. Malviya G, Galli F, Sonni I, Signore A. Imaging T-lymphocytes in inflammatory diseases: a nuclear medicine approach. Q J Nucl Med Mol Imaging. 2014;58: 237-257.

8. Moroz MA, Zhang H, Lee J, et al. Comparative analysis of T cell imaging with human nuclear reporter genes. $J$ Nucl Med. 2015;56:1055-1060.

9. Lopes FP, de Azevedo MN, Marchiori E, da Fonseca LM, de Souza SA, Gutfilen B. Use of ${ }^{99 \mathrm{~m}} \mathrm{Tc}$-anti-CD3 scintigraphy in the differential diagnosis of rheumatic diseases. Rheumatology (Oxford). 2010;49:933-939.

10. Signore A, Glaudemans AW. The molecular imaging approach to image infections and inflammation by nuclear medicine techniques. Ann Nucl Med. 2011;25:681-700.

11. Tavaré R, McCracken MN, Zettlitz KA, et al. Immuno-PET of murine T cell reconstitution postadoptive stem cell transplantation using anti-CD4 and antiCD8 cys-diabodies. J Nucl Med. 2015;56:1258-1264.

12. Venneti S, Lopresti BJ, Wiley CA. Molecular imaging of microglia/macrophages in the brain. Glia. 2013;61:10-23.

13. Owen DR, Yeo AJ, Gunn RN, et al. An 18-kDa translocator protein (TSPO) polymorphism explains differences in binding affinity of the PET radioligand PBR28. J Cereb Blood Flow Metab. 2012;32:1-5.

14. Zanotti-Fregonara $P$, Zhang $Y$, Jenko KJ, et al. Synthesis and evaluation of translocator $18 \mathrm{kDa}$ protein (TSPO) positron emission tomography (PET) radioligands with low binding sensitivity to human single nucleotide polymorphism rs6971. ACS Chem Neurosci. 2014;5:963-971.

15. Haugh O, Penman J, Irving AJ, Campbell VA. The emerging role of the cannabinoid receptor family in peripheral and neuro-immune interactions. Curr Drug Targets. 2016;17:1-8.

16. Savonenko AV, Melnikova T, Wang Y, et al. Cannabinoid CB2 receptors in a mouse model of Abeta amyloidosis: immunohistochemical analysis and suitability as a PET biomarker of neuroinflammation. PLoS One. 2015;10:e129618.

17. Esposito G, Giovacchini G, Liow JS, et al. Imaging neuroinflammation in Alzheimer's disease with radiolabeled arachidonic acid and PET. J Nucl Med. 2008;49:1414-1421.

18. Ji B, Kumata K, Onoe H, et al. Assessment of radioligands for PET imaging of cyclooxygenase-2 in an ischemic neuronal injury model. Brain Res. 2013;1533: $152-162$.

19. Nighoghossian N, Wiart M, Cakmak S, et al. Inflammatory response after ischemic stroke: a USPIO-enhanced MRI study in patients. Stroke. 2007;38:303-307.

20. Tourdias T, Roggerone S, Filippi M, et al. Assessment of disease activity in multiple sclerosis phenotypes with combined gadolinium- and superparamagnetic iron oxide-enhanced MR imaging. Radiology. 2012;264:225-233.

21. Moore KJ, Tabas I. Macrophages in the pathogenesis of atherosclerosis. Cell. 2011;145:341-355.

22. Ali A, Tawakol A. FDG PET/CT imaging of carotid atherosclerosis. Neuroimaging Clin N Am. 2016;26:45-54.

23. Menezes LJ, Kotze CW, Agu O, et al. Investigating vulnerable atheroma using combined ${ }^{18} \mathrm{~F}$-FDG PET/CT angiography of carotid plaque with immunohistochemical validation. J Nucl Med. 2011;52:1698-1703.
24. Foss CA, Bedja D, Mease RC, et al. Molecular imaging of inflammation in the ApoE -/- mouse model of atherosclerosis with IodoDPA. Biochem Biophys Res Commun. 2015;461:70-75.

25. Gaemperli O, Shalhoub J, Owen DR, et al. Imaging intraplaque inflammation in carotid atherosclerosis with ${ }^{11} \mathrm{C}$-PK11195 positron emission tomography/computed tomography. Eur Heart J. 2012;33:1902-1910.

26. Malmberg C, Ripa RS, Johnbeck CB, et al. ${ }^{64} \mathrm{Cu}$-DOTATATE for noninvasive assessment of atherosclerosis in large arteries and its correlation with risk factors: head-to-head comparison with ${ }^{68} \mathrm{Ga}$-DOTATOC in 60 patients. $\mathrm{J} \mathrm{Nucl}$ Med. 2015;56:1895-1900.

27. Bala G, Blykers A, Xavier C, et al. Targeting of vascular cell adhesion molecule1 by ${ }^{18} \mathrm{~F}$-labelled nanobodies for PET/CT imaging of inflamed atherosclerotic plaques. Eur Heart J Cardiovasc Imaging. January 22, 2016 [Epub ahead of print].

28. Morishige K, Kacher DF, Libby P, et al. High-resolution magnetic resonance imaging enhanced with superparamagnetic nanoparticles measures macrophage burden in atherosclerosis. Circulation. 2010;122:1707-1715.

29. Millon A, Dickson SD, Klink A, et al. Monitoring plaque inflammation in atherosclerotic rabbits with an iron oxide (P904) and ${ }^{18}$ F-FDG using a combined PET/MR scanner. Atherosclerosis. 2013;228:339-345.

30. Stendahl JC, Sinusas AJ. Nanoparticles for cardiovascular imaging and therapeutic delivery, part 2: radiolabeled probes. J Nucl Med. 2015;56:1637-1641.

31. Abdulla S, Salavati A, Saboury B, Basu S, Torigian DA, Alavi A. Quantitative assessment of global lung inflammation following radiation therapy using FDG PET/CT: a pilot study. Eur J Nucl Med Mol Imaging. 2014;41:350-356.

32. Amin R, Charron M, Grinblat L, et al. Cystic fibrosis: detecting changes in airway inflammation with FDG PET/CT. Radiology. 2012;264:868-875.

33. Harris RS, Venegas JG, Wongviriyawong C, et al. ${ }^{18} \mathrm{~F}-\mathrm{FDG}$ uptake rate is a biomarker of eosinophilic inflammation and airway response in asthma. $\mathrm{J} \mathrm{Nucl}$ Med. 2011;52:1713-1720.

34. Huang HJ, Isakow W, Byers DE, et al. Imaging pulmonary inducible nitric oxide synthase expression with PET. J Nucl Med. 2015;56:76-81.

35. Withana NP, Ma X, McGuire HM, et al. Non-invasive imaging of idiopathic pulmonary fibrosis using cathepsin protease probes. Sci Rep. 2016;6:19755.

36. Alam SR, Shah AS, Richards J, et al. Ultrasmall superparamagnetic particles of iron oxide in patients with acute myocardial infarction: early clinical experience. Circ Cardiovasc Imaging. 2012;5:559-565.

37. Bierry G, Lefevre S, Dietemann JL, Jehl F. In vivo macrophage imaging using MR targeted contrast agent for longitudinal evaluation of septic arthritis. $J$ Vis Exp. 2013:e50296.

38. Bruijnen ST, Gent YY, Voskuyl AE, Hoekstra OS, van der Laken CJ. Present role of positron emission tomography in the diagnosis and monitoring of peripheral inflammatory arthritis: a systematic review. Arthritis Care Res (Hoboken). 2014;66:120-130.

39. Gent YY, Ter Wee MM, Voskuyl AE, et al. Subclinical synovitis detected by macrophage PET, but not MRI, is related to short-term flare of clinical disease activity in early RA patients: an exploratory study. Arthritis Res Ther. 2015;17:266.

40. Thurman JM, Serkova NJ. Non-invasive imaging to monitor lupus nephritis and neuropsychiatric systemic lupus erythematosus. F1000Res. 2015;4:153. 